We have since drawn up our own guidelines for the use of anticholinergics in patients on neuroleptics. These may be of interest to those working in similar settings and are as follows.

(a) Routine use is not recommended.

(b) At the onset of neuroleptic treatment where prescribing for in-patients, anticholinergics should only be used in the presence of acute dystonia and should be gradually withdrawn after one week.

(c) At the onset of neuroleptic treatment where prescribing for out-patients, anticholinergics should be given for two weeks where: (i) the patient is a younger male or a child; (ii) there is a past history of drug-induced dystonia; or (iii) the patient lives a considerable distance from psychiatric care.

(d) During longer-term neuroleptic treatment, anticholinergics should be prescribed only where Parkinsonism has developed and where reduction of neuroleptic dose has been ineffective. In these cases anticholinergics should be given gradually but withdrawn after two months. If symptoms persist it may be sufficient to prescribe anticholinergics strictly for one week after each depot injection. In a small number of patients with disabling bradykinesia, long-term anticholinergics will be needed in combination with the lowest possible dose of neuroleptic.

M. A. ABAS

S. W. ACUDA

J. C. BROADHEAD

I. V. ChagWEDERA

F. B. ChIKARA

J. M. Piachaud

M. STEFANOVIC

J. VeRMEULEN

Department of Psychiatry

University of Zimbabwe

PO Box $A 178$

Avondale

Harare, Zimbabwe

\section{Benefits of routine laboratory investigations}

SIR: White \& Barraclough (Journal, July 1989, 155, 65-72) concluded that only very limited routine laboratory testing was justified for adult psychiatric admissions. We examined the results of laboratory screening tests, ordered on admissions to our inpatient inner-city general psychiatry unit, to determine the frequency of abnormal results and their clinical significance, and compared our results with those of Drs White \& Barraclough. The patient population was primarily male veterans with substance use, psychotic and major mood disorders. A total of 2308 tests on 52 admissions of 50 patients were examined. Each admission had an average of 44.4 tests and 5.5 abnormalities; each patient had at least one abnormal result. A total of $288(12.5 \%)$ of the tests ordered were abnormal (a figure not unlike the $10.2 \%$ reported by White \& Barraclough). No unexpected physical condition responsible for psychiatric symptoms was detected by the tests.

Of the abnormal results, 26 were positive toxicology screens in 20 patients with an already known substance use diagnosis (and were hence not unexpected results), and four were from a delirious patient with known chronic renal failure. A substance use diagnosis (including alcohol use disorders) accounted for 80 of the abnormal tests, including not unexpected elevations in liver enzymes, macrocytosis, elevated mean corpuscular haemoglobin concentration, bilirubin and low albumin, platelets or folate. Eleven abnormalities seemed to be related to poor nutrition (e.g. ketonuria on urinalysis, decreased blood urea nitrogen and creatinine), and 30 seemed to be associated with dehydration. Eighteen abnormalities were in AIDS patients and were important in monitoring their azidothymidine treatment. One psychotic patient was slightly hyponatraemic, probably secondary to psychogenic polydipsia. The remainder of the abnormalities were classified as without clear significance or diagnostic relevance.

We agree with Drs White \& Barraclough that most of the abnormalities were predictable and do not appear helpful in establishing previously unsuspected organic causes for psychiatric symptoms. However, $170(60 \%)$ of them were felt to be clinically significant (i.e., useful in treatment planning), as they: (a) had utility as a gauge of the severity of the patient's psychiatric condition (e.g. dehydration in a psychotic or depressed patient or liver damage in an alcohol abuser); (b) were useful in confronting the denial commonly seen in alcohol and substance abusers (e.g. using elevated liver enzymes in an alcoholic, positive toxicology screen results in a known drug-abuser denying recent use); (c) were useful in the detection of use of drugs other than those acknowledged by the patient; or (d) were important in managing various organic medical and psychiatric therapies. We would add these to the list of laboratory test benefits outlined by Drs White \& Barroclough. On the basis of our study, we agree with Drs White \& Barraclough's suggestion for only a selective laboratory screen on admission. The only aspect of laboratory screening on admission that should be 'routine' 
for the experienced psychiatric clinician is the informed, rational use of any test that is ordered.

PETER KAMP

RICHARD B. ROSSE

Washington Veterans Administration Medical Center Psychiatry Service

50 Irving Street $N W$

Washington, DC, 20422

\section{Dyskinesia in the mentally handicapped}

SIR: It was very interesting to read the well designed study of a well defined population by Dinan \& Golden (Journal, July 1990, 157, 131-132).

We have undertaken the screening of the whole mentally handicapped population at Cell Barnes Hospital $(n=550)$ for dyskinesia. After an extensive trial period we decided to use the Dyskinesia Identification System Condensed User Scale (DISCUS) instead of the Abnormal Involuntary Movement Scale (AIMS) because of the former's superiority in identifying facial tics and grimaces, and in having separate items for the tongue movements, tongue thrust, tongue tremor and athetoid/myokymic/ lateral tongue (Sprague et al, 1989). Also, DISCUS is a standardised and validated scale for mentally handicapped people who offer little or no co-operation.

In our pilot study, the prevalence of orofacial dyskinesia in the institutionalised mentally handicapped population was $77 \%$, in accordance with Dinan \& Golden's results. Richardson et al (1986) found that their group of mentally handicapped people with tardive dyskinesia was characterised by a $92 \%$ rate of buccolingual masticatory movements.

We believe that large multicentre trials on mentally handicapped people with various psychiatric and drug histories, comparisons of various dyskinesia scores, and observations of affective changes in relation to dyskinesia should be the next step forward in the attempt to elucidate the pathophysiology of dyskinesia.

\section{Charing Cross/Westminster Medical School \\ Unit of Mental Handicap}

D. KOHEN

London W6

Cell Barnes Hospital

G. MATHEW

Highfield Lane

St Albans, Hertfordshire AL4 ORG

\section{References}

Richardson, M. A., Haugland, G., Pass, R., et al (1986). The prevalence of Tardive Dyskinesia in mentally retarded population. Psychopharmacology Bulletin, 22, 243-249.
Sprague, R. L., Kalachinik, J. E. \& Shaw, K. M. (1989) Psychometric properties of the Dyskinesia Identification System: Condensed User Scale (DISCUS). Mental Retardation, 27, 141-148.

\section{Linguistics of schizophrenia}

SIR: Thomas et al (Journal, February 1990, 156, 204 210) used methods of linguistic analysis to examine the free speech of schizophrenics and others, finding that three of the 16 descriptive variables which discriminated between clinical groups were directly concerned with 'embedding'. In an experimental study designed to investigate the psychological processes involved in the comprehension of sentences, Huff and I systematically varied 'degree of embedding' to generate 12 sentence-types with varying degrees of difficulty (Thomas \& Huff, 1971).

Each sentence specified the rooms occupied by four people (Doctor, Grocer, Lawyer, Teacher) in a house containing six numbered rooms, two on each floor. While a sentence was displayed, the subject had to put dummy 'people' into an up-ended box with six compartments so as to depict its meaning. The time taken by the subject to "do his best" (T) and the number of 'people' correctly placed $(C)$ were averaged for each sentence-type, for two groups of subjects separately and combined. The subjects were eight young adults with acute schizophrenic or schizophrenia-like illnesses and eight normal young adult controls.

High negative correlations $(-0.85$ to -0.95 , $P<0.001$ ) were consistently found between $\mathrm{C}$ and $\mathrm{T}$. This relationship was linear and essentially the same for patients and controls. The rank-order of difficulty for the sentence-types, with either $C$ or $T$ as criterion, was similar for the two groups. Evidently a single aspect of sentence structure was causing most of the difficulty, in broadly similar ways for patients and normals, although the patients performed much worse than the normals.

As the effect of 'embedding' is to make a sentence more parenthetical, we next assumed that different sentence structures imposed different amounts of 'parenthetical load' (PL) on the subject by making greater or smaller demands on the decoding processes required to extract their meaning; and secondly, that patients were more vulnerable to PL than normals. Various assumptions could be made as to the nature of the decoding processes, and each combination of assumptions (each 'model') dictated its own procedure for calculating the $\mathrm{PL}$ of any given sentence. It was consistently found that $\mathrm{C}$ correlated negatively and T positively with PL; but one model was significantly 'better' than the rest, i.e. it gave 